Article

\title{
MISF2 Encodes an Essential Mitochondrial Splicing Cofactor Required for nad2 mRNA Processing and Embryo Develop- ment in Arabidopsis thaliana
}

\author{
Tan-Trung Nguyen ${ }^{1,+}$, Corinne Best ${ }^{2,+}$, Sofia Shevtsov ${ }^{2}$, Michal Zmudjak ${ }^{2}$, Martine Quadrado ${ }^{1}$, Ron Mizrahi ${ }^{2}$, \\ Hagit Zer ${ }^{2}$, Hakim Mireau ${ }^{1, *}$ and Oren Ostersetzer-Biran ${ }^{2, *}$ \\ 1 Institut Jean-Pierre Bourgin INRAE, AgroParisTech, CNRS, Université Paris-Saclay, Versailles, France \\ 2 Department of Plant and Environmental Sciences, The Alexander Silberman Institute of Life Sciences, The \\ Hebrew University of Jerusalem, Givat-Ram, Jerusalem 91904, Israel \\ $\dagger$ These authors have equally contributed to the MS. \\ * Correspondence: Hakim Mireau, E-mail, hakim.mireau@versailles.inra.fr, and Oren Ostersetzer-Biran. E- \\ mail, oren.ostersetzer@mail.huji.ac.il.
}

\begin{abstract}
Mitochondria play key roles in cellular energy metabolism in eukaryotes. Mitochondria of most organisms contain their own genome and specific transcription and translation machineries. The expression of angiosperm mtDNA involves extensive RNA-processing steps, such as RNA trimming, editing, and the splicing of numerous group II-type introns. Pentatricopeptide repeat (PPR) proteins are key players of plant organelle gene expression and RNA metabolism. In the present

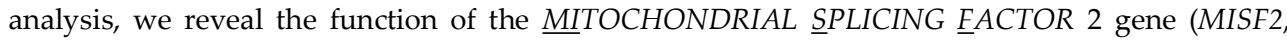
AT3G22670) and show that it encodes a mitochondria-localized PPR protein that is crucial for early embryo-development in Arabidopsis. Molecular characterization of embryo-rescued misf2 plantlets indicates that the splicing of nad 2 intron 1 and thus respiratory complex I biogenesis are strongly compromised. Moreover, the molecular function seems conserved between MISF2 protein in Arabidopsis and its orthologous gene (EMP10) in maize, suggesting that the ancestor of MISF2/EMP10 was recruited to function in nad2 processing before the monocot-dicot divergence, $\sim 200$ million years ago. These data provide new insights into the function of nuclear-encoded factors in mitochondrial gene expression and respiratory chain biogenesis during plant embryo development.
\end{abstract}

Keywords: group II; intron; splicing; PPR; respiration; complex I; mitochondria; embryogenesis; arabidopsis; angiosperms

\section{Introduction}

Mitochondria are key sites of cellular energy metabolism (i.e., ATP production), as well as for the biosynthesis of various essential metabolites. Most modern mitochondria contain vestigial genomes ( $\mathrm{mtDNA}$, mitogenome) derived from that of their ancestral bacterial progenitor, and which vary quite widely in size between organisms. In plants, angiosperm mtDNAs are remarkably large and complex in structure [1], encoding rRNAs, tRNAs, ribosomal proteins, as well as various subunits of respiratory complexes (CI to $\mathrm{CIV})$, the ATP synthase enzyme (CV), cofactors of the cytochrome c biogenesis (CCM) machinery, and at least one component of the twin-arginine protein translocation system [2].

In Arabidopsis, the oxidative phosphorylation (OXHPOS) machinery is composed of $>100$ different subunits, most of which are encoded by nuclear loci and about 20 are expressed from the mitogenome. The biogenesis of the respiratory chain machinery thus involves various mechanisms for regulating the expression of subunits that are encoded 
by two physically separate genetic compartments [3-5]. However, the identity of factors and pathways involved in these regulations remain largely elusive.

The expression of mitochondrial genes in plants involves extensive RNA-processing steps. These include RNA trimming, RNA editing, and the removal of many intron sequences that reside in various genes [6-9]. These RNA processing steps are essential for mt-RNAs to synthesize the protein they encode. While the excision of canonical group II introns rely on proteins encoded by the introns themselves (i.e., IEPs, or maturases) [10, $11]$, the splicing of the plant organellar group II introns involves a repertoire of nuclearencoded factors that assist splicing reactions and which may serve as key control points in plant mitochondrial gene expression [1, 12-14]. These belong to diverse families of RNA binding factors. A few are related to maturases [14, 15], whereas others were identified as e.g. RNA helicases [16-18], PORR-related proteins [19], and relevantly to our study also pentatricopeptide repeat (PPR) proteins [20].

The PPR family constitutes a large protein family in land plants, with approximately 450 members identified in Arabidopsis and 490 genes in maize [21-23]. PPR proteins are recognized by a degenerate 35 amino-acid motif folding into two antiparallel helices connected by a short loop/turn [24, 25]. PPRs have been shown to play multifarious functions in organellar RNA metabolism, such as RNA stability and protection [8, 26], C-to-U RNA editing [9], mRNA translation (Haili et al 2016, Waltz et al, 2019, Nguyen et al, 2021) and group II intron splicing [6, 8, 27-29]. Members of the PPR family are also linked to fertility restoration, where they regulate the expression of mitochondrial CMS-associated ORFs $[30,31]$.

Genetic and biochemical studies indicated that PPRs are sequence-specific RNAbinding trans-factors, and that RNA recognition is mostly mediated by amino acids found at positions 5 and 35 in each PPR repeat. Association with each of the four RNA bases involves specific amino acid combinations that are the basis of a PPR protein-RNA recognition code $[32,33]$. These data were further supported by the analysis of PPR proteinRNA crystal structures [24, 25, 34-37]. PPRs are classified into two main groups: P and PLS type proteins, which in addition to canonical 35-amino acid PPR motifs (P) include long (L) or short $(S)$ repeat variants $[22,38]$. While PLS-type proteins are almost exclusively associated with RNA editing [9, 39], P-type PPR factors facilitate a wide range of organellar RNA expression steps going from stabilization to translation [27, 40]. In this work, we analyzed the function of a mitochondrial P-type PPR factor that we named $M I$ TOCHONDRIAL INTRON SPLICING FACTOR 2 (MISF2). The Arabidopsis MISF2 protein is homologous to the PPR protein EMP10 in Zea mays [41]. We show that MISF2 is required for the splicing of nad2 intron 1, and that the respiratory complex I biogenesis is strongly destabilized in misf2 mutants. The molecular function of MISF2 is conserved in both Arabidopsis and maize [41], suggesting that the common ancestor MISF2 was recruited to function in nad2 splicing prior to the divergence of monocot and dicot plants, i.e. about 200 million years ago [42].

\section{Results}

\subsection{The topology of MISF2 protein}

To better understand processes associated with mitochondrial RNA (mt-RNA) expression in plants, we assembled a collection of Arabidopsis T-DNA mutants affected in genes encoding mitochondria-targeted P-type PPR proteins and identified that heterozygous plants carrying insertions in the At3g22670 gene could not set homozygous mutants in their progeny. Domain search analysis using the PPRfinder [43], PPRCODE [33], SMART [44] and CDD [45] algorithms indicated that the deduced product of AT3G22670 gene (Figure 1, Supplementary Figure S1) encodes a 562 amino acid PPR protein with a predicted topology of $\mathrm{NH}_{2}-165-\mathrm{P}-3-\mathrm{P}-\mathrm{P}-\mathrm{P}-\mathrm{P}-\mathrm{P}-\mathrm{P}-\mathrm{P}-\mathrm{P}-\mathrm{P}-42-\mathrm{COOH}$ (where ' $\mathrm{P}$ ' designates Ptype PPR motifs and amino acids not assigned to any defined domain are specified by numbers) (Figure 1, Figure S1a). 
Subcellular localization prediction algorithms, available at the ExPASy portal (https://www.expasy.org), UniProt [46] and the 'SUBcellular location database for Arabidopsis proteins' (SUBA4, http://suba.live) [47], indicate the presence of a predicted 24amino acid mitochondrial targeting sequence in the N-terminal region of MISF2 (Figure S1a). In silico 3D structure prediction, using the AlphaFold server [48], suggested that MISF2 harbors a typical PPR helical fold (Figure S1a), with an inner basic core representing the RNA binding surface, as previously indicated from the analysis of the plant PPR10 protein [25].

a

At3g22680 (RDM1)

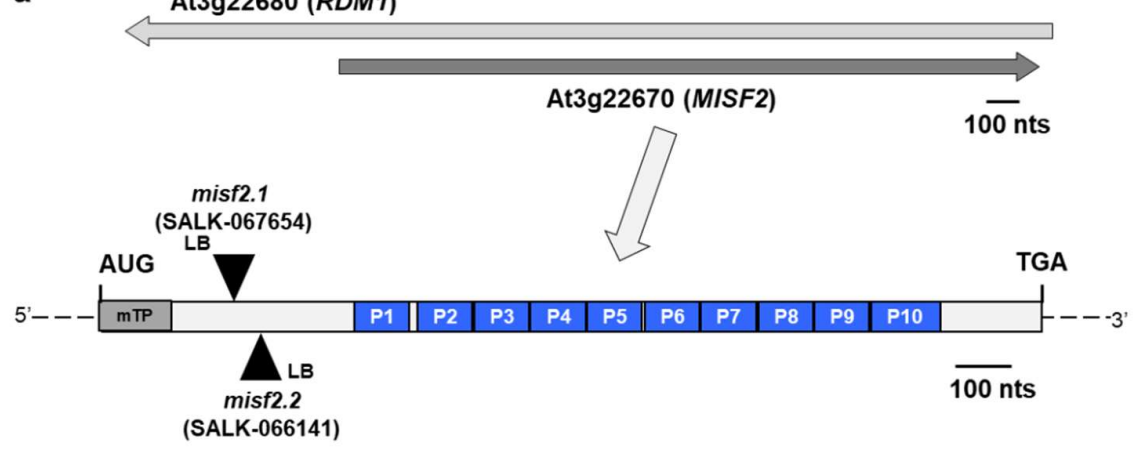

b
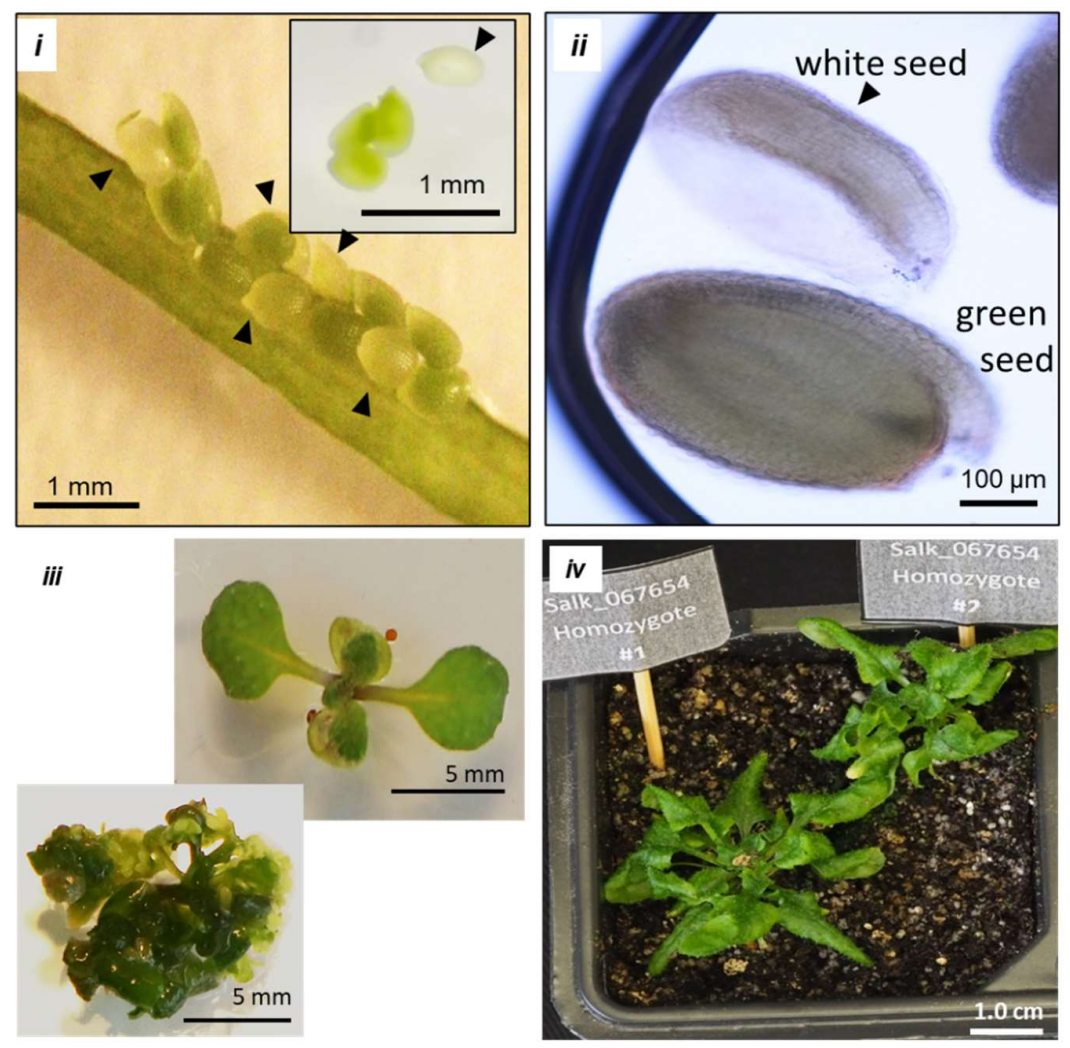

Figure 1. MISF2 (At3g22670) gene topology and misf2 mutant phenotypes. (a) Scheme of the At3g22670 locus and gene structure. The position of the two T-DNA insertion sites in the coding region of MISF2 (i.e., SALK-line 067654, misf2.1 and SALK-line 066141, misf2.2) are located 324 and 350 nucleotides downstream to the ATG start codon and which correspond to the N-terminal region, upstream to the PPR motifs of the MISF2 protein. (b) Morphologies of misf2 mutants. Green (wild type or heterozygous) and white (homozygous) seeds were collected from surface-sterilized immature siliques of heterozygous misf2 plants $(i)$ and sown on MS agar media supplemented with 
vitamins plants. Arrows point toward white seeds. Panel B-ii shows differential interference contrast microscopy images (i.e., Nomarski) of the embryos found in green or white seeds. Following germination, few rescued misf2 plantlets (iii) were able to survive on soil, although failed to flower and set viable seeds $(i v)$.

\subsection{MISF2 encodes a lowly-expressed P-type PPR protein that is localized in mitochondria}

Expression analysis of MISF2 were made using publicly available microarray and high-throughput sequencing databases. The Arabidopsis Information Resource (TAIR) (http://www.arabidopsis.org) and 'Genevestigator analysis toolbox' [49] databases indicated differential expression of MISF2 gene throughout development, with MISF2 expression being dominant in embryonic organs, young developing leaves, apical root tissues, flowers and the shoot apex (Figure S2). To further investigate the intracellular location of MISF2, a fragment comprising the first 203 amino acids of MISF2 was fused in-frame to GFP (MISF2-GFP), expressed in Arabidopsis plants and the subcellular localization of the resulting fluorescence examined by confocal microscopy (Figure 2). In agreement with the in-silico data, the MISF2-GFP signal was detected as round-shaped particles that co-localized with those of the MitoTracker ${ }^{\circledR}$ marker, a mitochondrion-specific fluorescent probe (Figure 2). These results are consistent with the predicted mitochondrial targeting of MISF2.
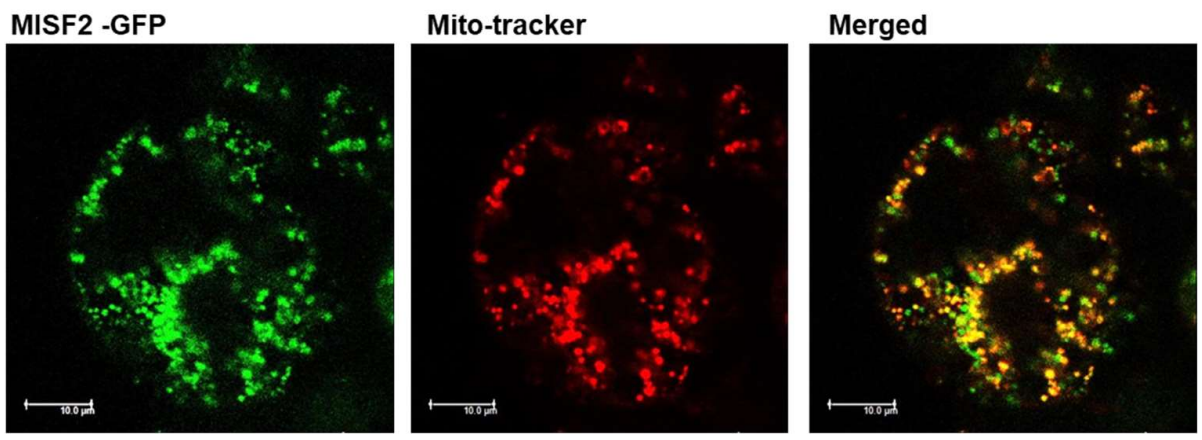

Figure 2. MISF2 is localized to the mitochondria. Arabidopsis plant cells were transformed with a construct expressing the GFP fused in frame to the N-terminal region (i.e., 203 amino acids) of the Arabidopsis MISF2 protein sequence. The fluorescence corresponding to the GFP (green, left), the MitoTracker ${ }^{\circledR}$ marker (red, center) and the merged signals (right) are shown.

\subsection{MISF2 is required for early embryo development in Arabidopsis thaliana}

Two T-DNA insertion lines were identified within the MISF2 gene. The SALK_067654 (misf2.1) and SALK_066141 (misf2.2) contain T-DNA insertions located 324 and 350 nucleotides downstream of MISF2 translational start, respectively (Figure 1a, and Figure S3a). No homozygous plants could be recovered from the progeny of heterozygote misf2 lines, suggesting that the At3g22670 gene is an essential gene that is critical during embryogenesis. Heterozygous misf2.1 and misf2.2 mutant lines did not show any obvious phenotypes under normal growth conditions (see Material and Methods) suggesting that homozygous mutants could be embryonic lethal. To test this assumption, we compared the developmental phenotypes of embryos contained in immature seeds (10 days after pollination) of heterozygous misf2 and wild type plants. Siliques of heterozygous misf 2 plants contained about one-quarter of yellow to white seeds (Figure 1b-i), which later degenerated into shrunken and brown mature seeds. Microscopy analyses further indicated that green seeds in siliques of heterozygous misf2 plants contained fully developed embryos, while white seeds had embryos arrested at the late torpedo/walking stick stages (Figures $1 \mathrm{~b}-i$ and $1 \mathrm{~b}-i i)$. 


\subsection{MISF2 is essential for nad2 pre-mRNAs processing in Arabidopsis mitochondria}

Although misf 2 is not found among the 32 Arabidopsis embryo-defective $p p r$ mutants of the 'SeedGene' database [50], our genetic and microscopic analyses indicate that MISF2 is essential for proper embryo development (Figure 1b). Embryo rescue approaches enable the establishment of certain Arabidopsis mutants showing germination-defective phenotypes [51]. Among these are a few mutants affected in mitochondria biogenesis and function, such as the cod1 [52], ndufv1 [53] cal1/cal2 [54, 55] or nmat3 mutants [56]. Therefore, white seeds contained in young siliques of heterozygous misf2 plants (i.e., 10 12 days post-anthesis, DPA), were sown on MS-agar plates supplemented with $1 \%$ sucrose and various vitamins (see Materials and Methods) and then transferred to controlled growth chamber. Indeed, under these conditions, 30\% of the white seeds have germinated after 3 months of culture and were then transferred to liquid culture using the same medium (see Materials and Methods). PCR genotyping indicated that while green seeds derived from misf2.1 or misf2.2 heterozygote plants were either wild type or heterozygous for the mutations, plantlets obtained from white seeds were all homozygous for either of the two misf2 mutant alleles.

The optimal conditions for rescue of misf2.1 seedlings were similar to those reported for the nmat3 mutant [56], whereas rescue of misf2.2 was achieved following the rescue method previously described for the Arabidopsis cod1 mutant [52]. Phenotypical variations between rescued misf2 plantlets were visible, as certain seedlings developed into slow-growing normal-looking plants with twisted leaves (Figure 1b-iii), while others produced miniature bushy-like structures (Figure 1b-iii), as previously reported for several other emb mutants [57], notably affected in mitochondria biogenesis [7, 52, 56]. Few homozygous misf2 plantlets (e.g., Figure 1b-iv) could be further transferred and cultivated on soil, but none could produce viable seeds. For RNA and protein analyses, we used 3week-old mutant plantlets [57]. MISF2 gene is partially overlapping with RDM1 (At3g22680) (Figure 1a), a nuclear DNA methyltransferase, which its functions in Arabidopsis are not essetial during embryogenesis [58]. To further establish the specific roles of MISF2 in mitochondria biogenesis, we generated a 'complemented' homozygous misf2.2 line expressing the native AtMISF2 gene (misf2.2/35S::MISF2).

The steady-state levels of mitochondrial mRNAs in misf2.1 and misf2.2 mutants were analyzed by RT-qPCR in comparison to wild type (Col-0) plants. This analysis revealed a strong reduction (i.e., about 70 to 1,200 folds) in the accumulation of mature nad 2 transcripts spliced from its first intron in misf2.1 and misf2.2 mutants, respectively (i.e., nad2 exons $a-b$, Figure 3a). The steady-state levels of most other mitochondrial transcripts, including nad2 transcripts spliced from its other introns were found to over-accumulate from 2 to 5 folds in both misf2 mutant lines (Figure 3a). The RNA profiles in plantlets derived from immature wild type embryos and grown under the same conditions as misf 2 mutants did not show any significant reductions in the accumulation of mitochondrial transcripts, including nad2 (Figure 3b). Similarly, the accumulation of nad2 transcripts in functionally complemented misf2.2 plants were globally equivalent to those in wild type plants (Figure $3 b$ and Figure S3b). Based on these data, we concluded that the maturation defects observed for nad2 transcripts in misf2.1 and misf2.2 plants relate to the functions of MISF2 and not to physiological differences between the embryo-rescued plantlets and 3week-old Arabidopsis seedlings germinated on MS-media plates. 

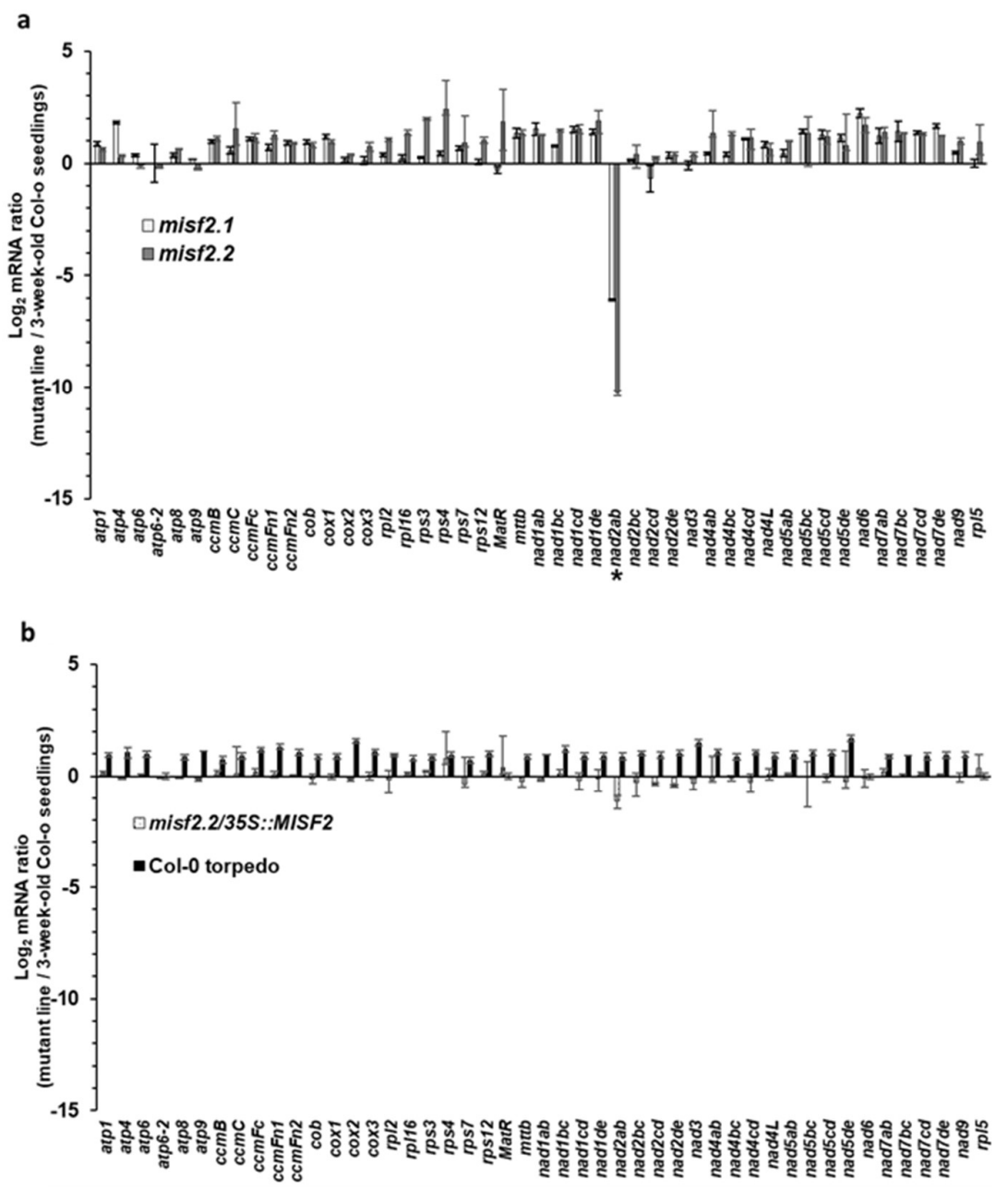

Figure 3. Relative accumulation of mitochondrial mRNAs in misf2 mutants. Transcriptome analyses of mt-RNAs levels in Arabidopsis wild type (Col-0) and misf2 mutant plants by RT-qPCR. RNA extracted from 3-week-old wild type seedlings (Col-0), 4-months-old rescued misf2 mutants, plantlets derived from immature Col-0 seeds (i.e., at the torpedo stage) and functionally complemented misf 2 mutants were reverse-transcribed and the relative steady-state levels of cDNAs corresponding to mitochondrial mRNAs evaluated by qPCR. $\log _{2}$ ratios of $\mathrm{mt}$ mRNA abundances in misf2.1 and misf2.2 mutant lines (a), plantlets derived from immature Col-0 seeds and complementation line (b) to those of 3-week-old MS-agar grown wild type plants. The values are means of three biological replicates (error bars indicate one standard deviation).

\subsection{MISF2 is required for efficient splicing of nad2 intron 1}

We reasoned that the reduced steady-state levels observed for the upstream region of mature nad2 transcripts (i.e., spliced exons ' $a$ ' and ' $b$ ') in misf2 mutants likely relate to defects in the excision of the first intron in nad2. We thus determined the splicing efficiencies of nad 2 intron 1 and that of the other 22 mitochondrial introns in wild type plants and germinated embryos, as well as in misf 2 mutant and functionally complemented misf 2.2 plants by RT-qPCR. The obtained data revealed a strong reduction in the splicing efficiency of nad2 intron 1, with splicing reductions reaching about 360 and 11,000 folds in misf2.1 and misf2.2 plants, respectively, compared with the wild type (Figure 4a). 

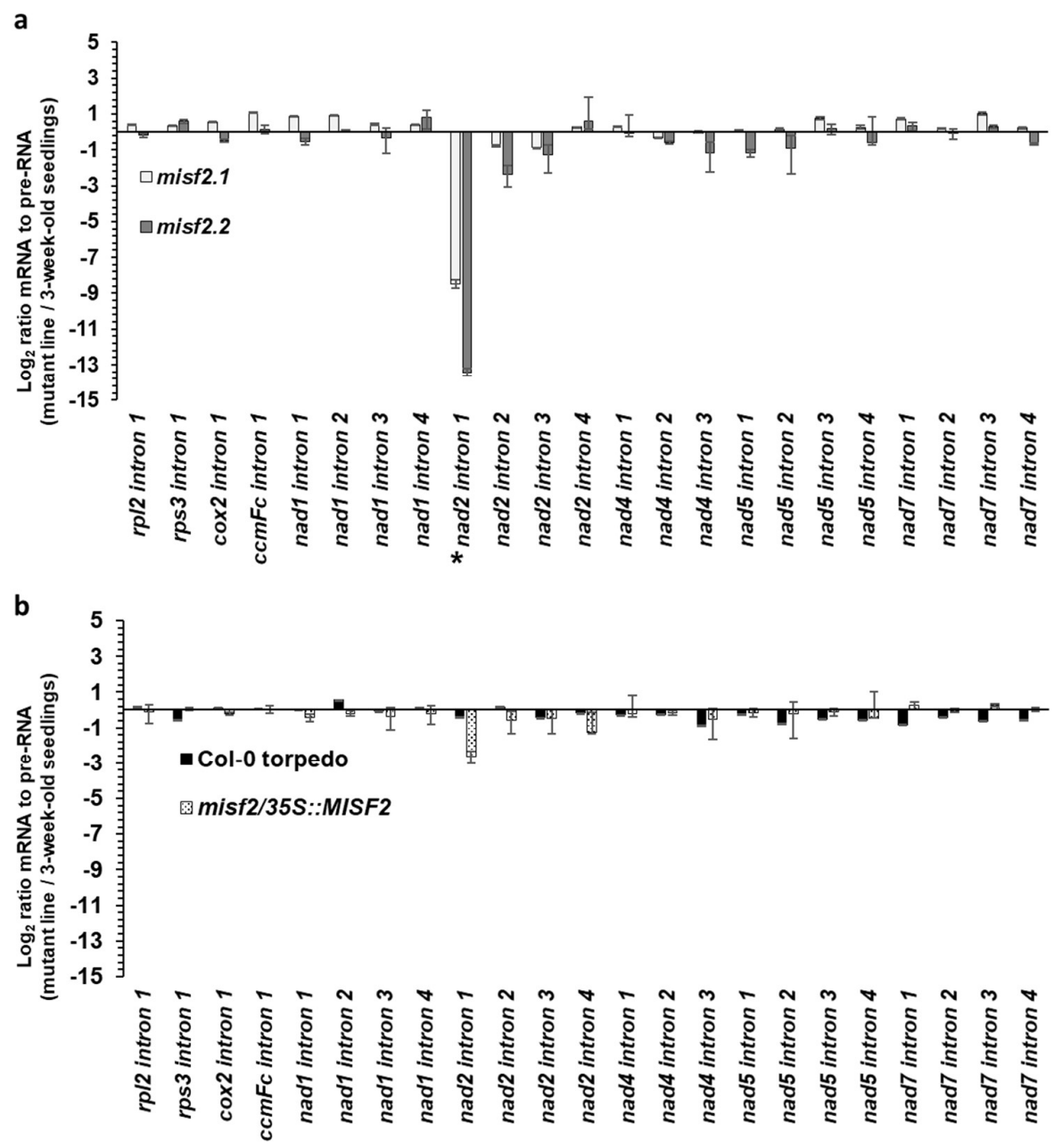

Figure 4. Splicing efficiencies in misf2 mutants. The relative accumulation of mRNA and pre-RNA transcripts in wild type and misf2 plants, corresponding to the 23 group II intron sequences in Arabidopsis, was evaluated by RT-qPCR. The histogram shows the splicing efficiencies as indicated by the $\log _{2}$ ratios of pre-RNA to mature mRNA transcript abundance in misf2-1 and misf2-2 mutant lines compared with those in wild type plants (a), as well as germinated wild type seeds collected at the torpedo stage (Col-0-torpedo) and complemented misf2-2 line compared with those of wild type plants (b). The values are means of three (misf2.1) and five (misf2.2) biological replicates (error bars indicate one standard deviation). .

In contrast to nad 2 intron 1 , the splicing efficiency of other mitochondrial transcripts was not significantly affected in misf2 mutants, although small reductions (i.e., 2.5 to 6.7 folds) in the splicing efficiencies of nad2 introns 2 and 3 were seen in misf 2.2 plants. The reduction in nad 2 intron 1 splicing observed in misf2 plants was largely corrected in complemented misf2.2 plants expressing the native AtMISF2 gene (misf2.2/35S::MISF2), strongly supporting the role for MISF2 in the processing of nad2 intron 1 pre-mRNA (Figure $4 b)$.

\subsection{The MISF2 protein associates with nad2 intron 1 in vivo}

PPR proteins are known to be sequence-specific RNA-binding factors [24, 27, 32, 5961]. A combinatorial code for RNA-recognition by PPR proteins was proposed, based on combinations of amino acids found at positions 5 and 35 of each PPR repeat [32, 61, 62]. Using this code for the prediction of binding sites for the 10 PPR repeats of MISF2 (Figure 1, Figure S1) suggested the following sequence: $5^{\prime}-N-(G>>A>U)-(U>C>G)-(U>C>G)-$ 
$(A>G)-(G>>A)-(G>>A>U)-(C>U)-G-N-3$ '. BLAST search along the updated Arabidopsis mtDNA (BK010421) revealed a matching 10-nucleotide sequence within domain I of nad2 intron 1 (Figure 5a).

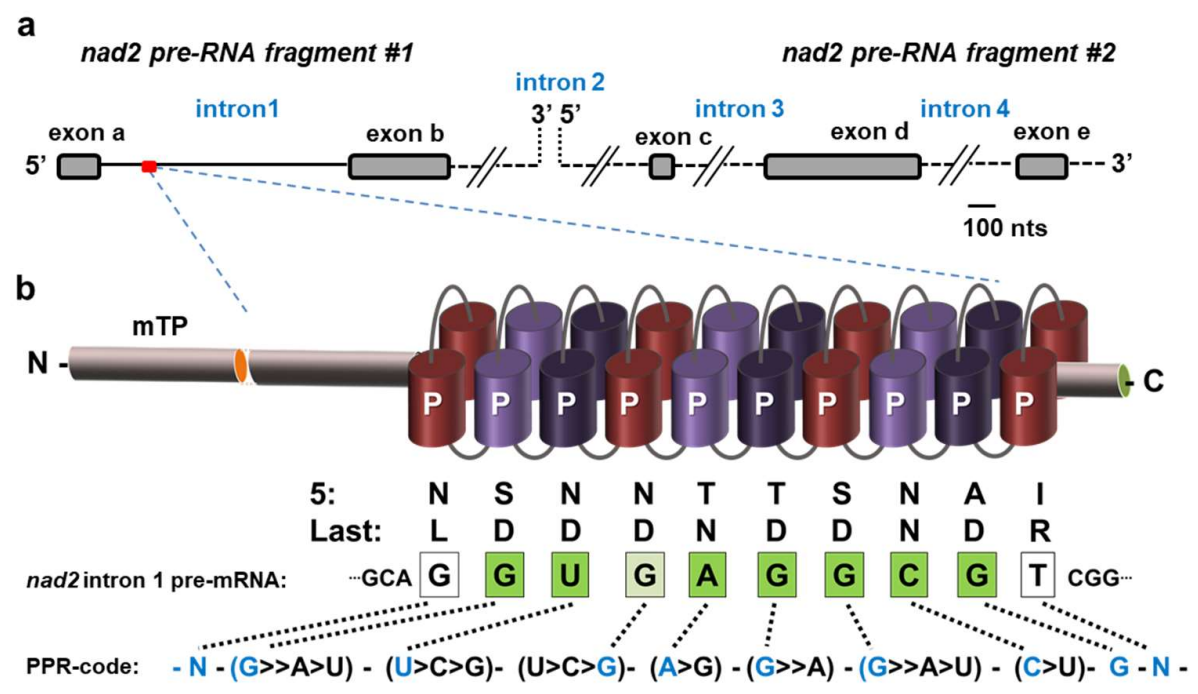

Figure 5. The predicted MISF2 binding site within domain I of nad2 intron 1. (a) The expression of nad2 in Arabidopsis mitochondria involves the transcription of two precursor RNA transcripts, which are divided by the second intron. The maturation of and mRNA requires the splicing of four group II introns found in both cis (nad2 introns 1, 3 and 4) and trans (intron 2) configurations. The first pre-RNA fragment consists of two exons separated by intron 1, while the second fragment harbors three exons separated by introns 3 and 4. (b) MISF2 is a P-type PPR protein, which harbors a mitochondrial localization signal (mTP) and 10 PPR motifs. The fifth and the last amino acids of each PPR repeat (Figure S1) are indicated below each PPR repeats. The best corresponding RNA binding site (i.e., 5' - GGUGAGGCGU - $3^{\prime}$ ) is indicated within the first intron of nad2 pre-RNA fragment \#1, with bases marked in green for perfect matches to the proposed binding site, in pale green for partial matches, and white for unassigned nucleotides.

No other sequences of 10 bases long corresponding to the predicted MISF2 binding site could be identified elsewhere in the plant mitogenome. A model for the asscoation of MISF2 with its predicted RNA bindng site within nad2 intron 1 is illustrated in (Figure $5 b)$. The in-silico data, therefore, correlated with the 'genetically defined' RNA target of MISF2, nad2 intron 1 (Figures 4 and 5).

To further examine the in vivo targets of MISF2, an HA-tagged version of MISF2 was expressed in Arabidopsis. After confirming the expression of the tagged protein in vivo (Figure 6a), the MISF2-3HA protein was immunoprecipitated from total extracts (Figure 6b) and co-purified RNAs were analyzed by RT-qPCR (Figure 6c). 
a

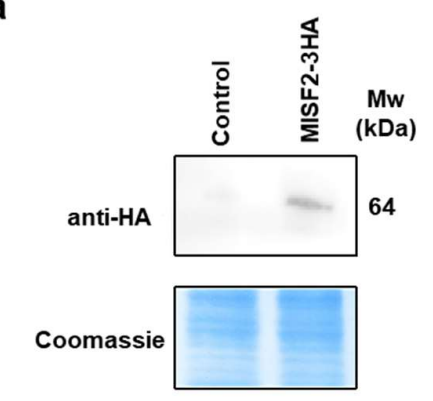

C

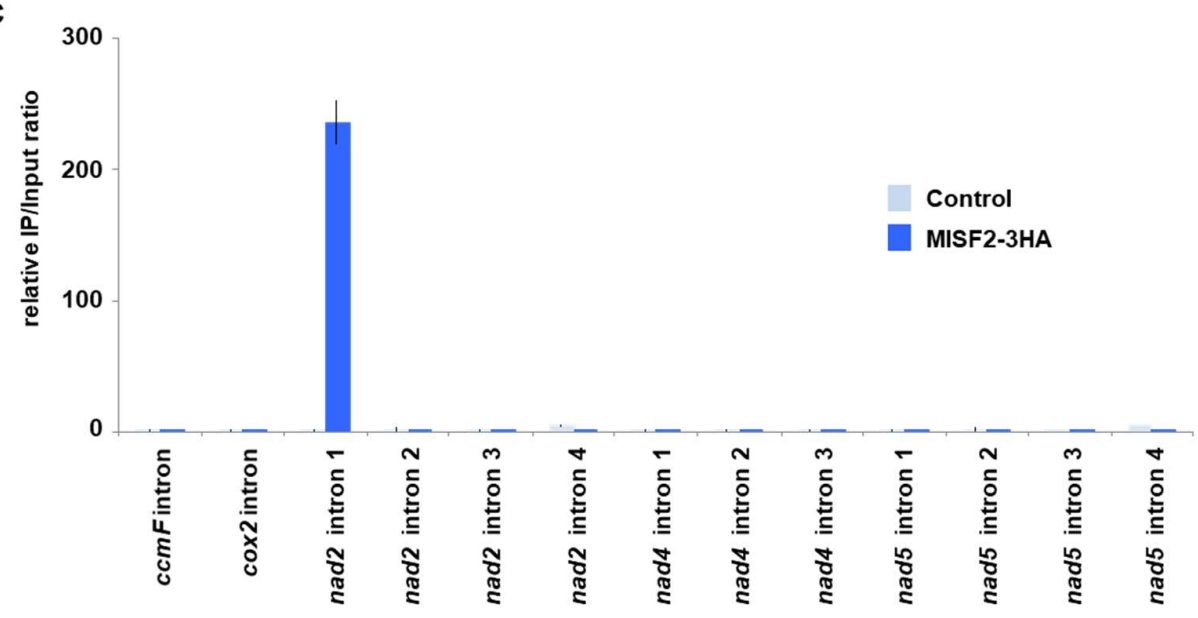

b

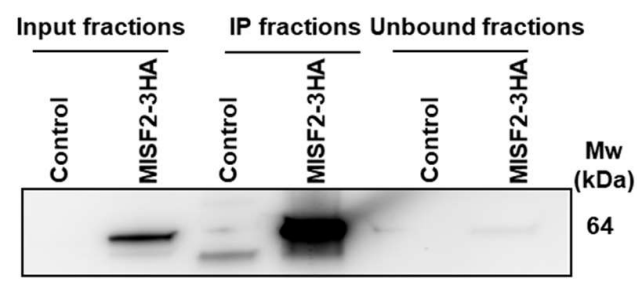

Coomassie

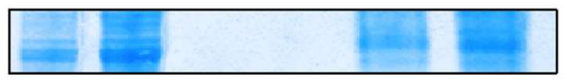

Figure 6. The MISF2 protein associates with nad2 intron 1 in vivo. (a) Immunodetection of the MISF2-3HA fusion protein in protein extracts prepared form untransformed (control) and transformed (MISF2-3HA) Arabidopsis cell cultures (b) Immunoprecipitation assays were conducted with the anti-HA antibody and the shown immunoblot analysis attests for the strong enrichment of the MISF2-3HA fusion in the immunoprecipitated (IP) fraction derived from the Arabidopsis transgenic cell line expressing the fusion. The weak MISF2-3HA signal in the unbound fraction demonstrates the efficiency of the immunoprecipitation. Parts of the blots stained with Coomassie blue are shown to display equal loading between samples. (c) Co-immunoprecipitated RNAs were analyzed by qRT-PCR using primer pairs specific to the indicated mitochondrial introns and relative enrichment ratios (immunoprecipitation fraction/input fraction) are shown.

Primers amplifying nad2 intron 1 was used in this analysis, along with other primers pairs targeting introns whose splicing was found to be slightly reduced in misf2 plants and additional controls. The obtained results revealed a very strong co-enrichment of nad 2 intron 1 specifically in the MISF2-3HA immunoprecipitate. None of the other tested introns (i.e., the single introns within $c c m F$ or cox 2 mRNAs, nad2 introns 2, 3 and 4, nad4 introns 1 to 3 , or nad5 introns 1 to 4 ) were co-enriched with MISF2-3HA, strongly supporting that nad2 intron1 is the in vivo RNA target of this PPR protein and thereby confirming that MISF2 specifically associates with its genetically defined intron RNA.

\subsection{Analysis of the biogenesis of the respiratory chain in misf2 mutants}

The respiratory system of plant cells is made of 5 major protein complexes, termed as complex I (CI, about 1,000 kDa in size), CII (160 kD), dimeric complex III (III2, $500 \mathrm{kDa})$, CIV (200 and $220 \mathrm{kDa}$ forms), and the ATP synthase (CV, $660 \mathrm{kDa}$ ) [63]. Plant mitochondria also harbor various enzymes that belong to the 'alternative electron transport' pathway, involving alternative NADH dehydrogenases and the alternative cytochrome oxydase [64]. Genetic and biochemical studies showed that Nad2 is essential for complex I (CI) biogenesis and function [65-72]. The reduction in nad2 splicing (Figures 3 and 4) suggests that the CI Nad2 subunit likely accumulates to very low levels in misf2 plants. Indeed, BN-PAGE analysis of Arabidopsis respiratory complexes indicated that $\mathrm{CI}$ is below 
detectable levels in misf2 mutant plants (Figure 7). Immunoblots made with antibodies against the carbonic anhydrase CA2 [73] further indicated the accumulation of several complex I assembly intermediates of about 610, 230 and $85 \mathrm{kDa}$ in both misf2 mutants. While CI was considerably reduced in both misf2 mutants, BN-PAGE analyses indicated that other respiratory complexes, including CIII, CV, and particularly CIV were rather upregulated in misf2 plants (Figure 7).
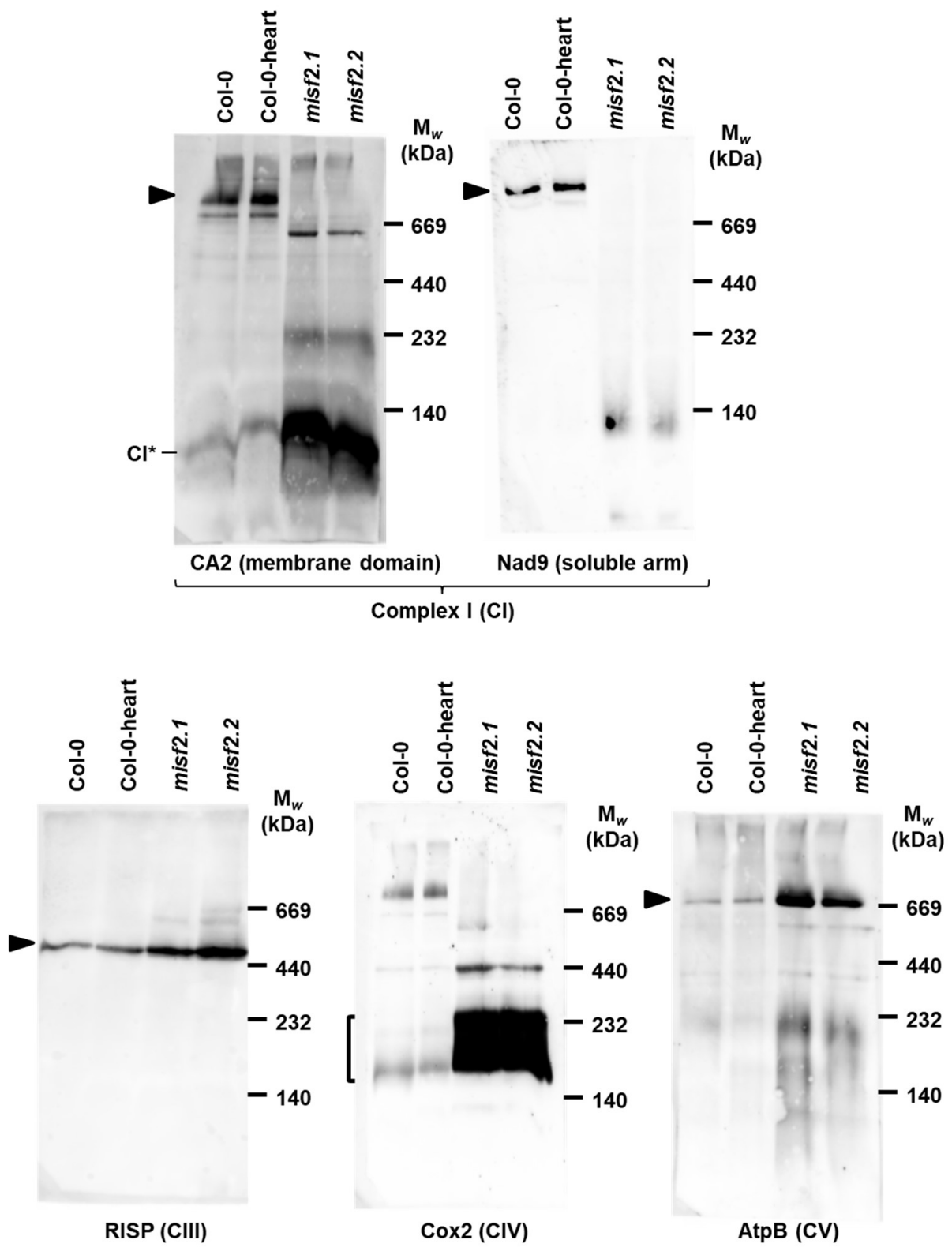

Figure 7. Holo-complex I is below detectable levels in misf2 mutants. Blue native (BN)-PAGE analysis of crude organellar fractions was performed as described by [74]. Aliquots, equivalent to $40 \mathrm{mg}$ of crude organellar membrane extracts, obtained from wild type and misf 2 plants, were solubilized with digitonin and resolved by BN-PAGE. For immunodetection, the proteins were transferred onto PVDF membranes and probed with the antibodies indicated below each blot (Table S2), as indicated. Arrows point toward native complexes I ( 1,000 kDa), CIII dimer (III2, $500 \mathrm{kDa}$ ), CIV (about 200 and $220 \mathrm{kDa}$ forms), and CV ( 660 kDa) [63]. CI* indicates the $\sim 85 \mathrm{kDa}$ sub-CI assembly intermediate [67]. 
We further analyzed the relative accumulation of various mitochondrial proteins in Col-0, misf2 mutants and the functionally-complemented misf2.2/35S::MISF2 line by immunoblotting analysis using various antibodies raised against different plant mitochondrial proteins. The data indicated that the CI-subunit CA2 and Nad9 accumulate in similar quantities in misf2 and wild type plants. The levels of various other mitochondrial proteins, including the Rieske iron-sulfur protein (RISP) of CIII, the Cox2 subunit of CIV, the AtpB subunit of $\mathrm{CV}$, and the mitochondrial outer-membrane voltage-dependent anion channel (VDAC, or PORIN) proteins were upregulated in misf2 mutants, as compared with the wild type plants (Figure 8a).

a

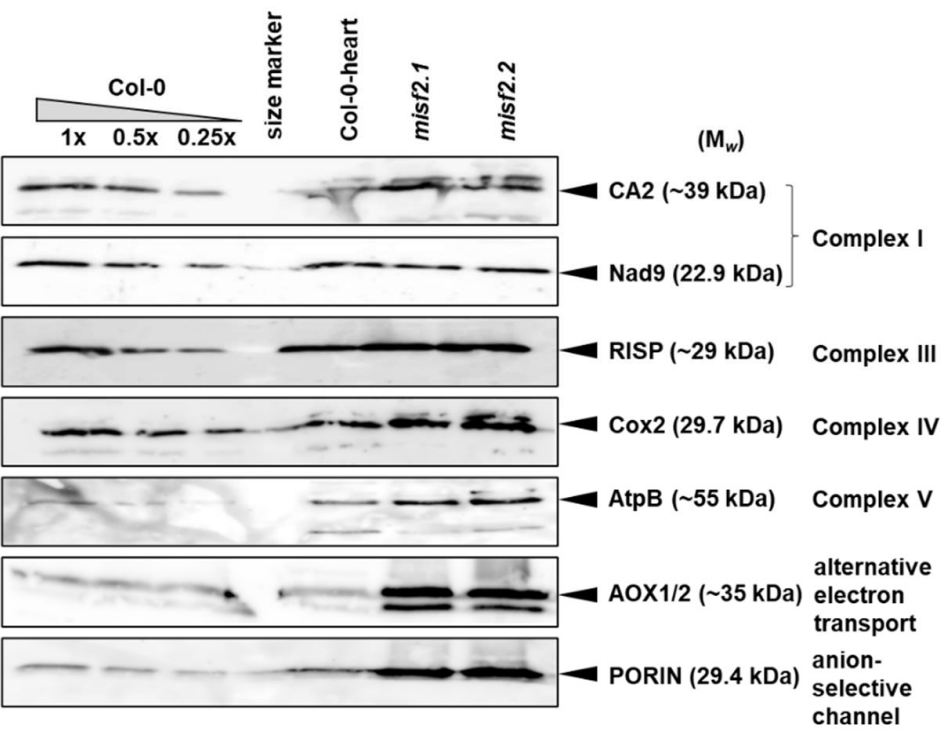

b

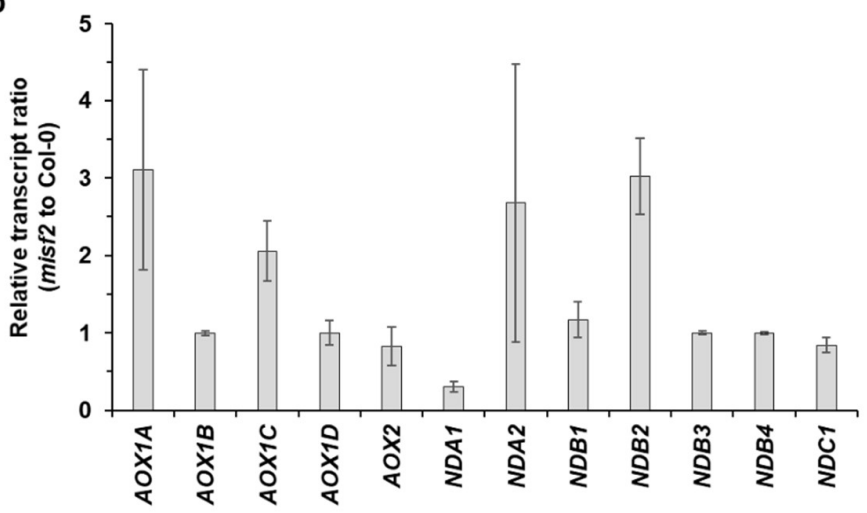

Figure 8. Relative accumulation of different mitochondrial proteins and $A O X$ or $N D$ transcripts in wild type and misf2 plants. (a) Immunoblots made with crude organellar fractions (equivalent to $\sim 40 \mathrm{mg}$ FW) extracted from 3-week-old MS-grown wild type plants, in vitro germinated wild type embyos at the heart to torpedo stages (Col-0 heart) and homozygous misf2 plantlets. The blots were probed with antibodies raised against the indicated mitochondrial proteins. (b) Analysis of the steady-state levels of various alternative oxidase (AOX) and rotenone-insensitive NAD(P)H dehydrogenase (ND) mRNAs by RT-qPCR. The histogram displays relative mRNAs levels in misf2 plantlets to 3-week-old MS-grown wild type plants.

In contrast, the accumulation of all tested mitochondrial proteins was equivalent between the complemented line (misf2.2/35S::MISF2) and wild type plants (Figure S4).

Arabidopsis mutants affected in CI biogenesis undergo oxidative stress and often show subsequently a strong induction of the alternative respiratory pathways $[53,55,72$, 75, 76]. Accordingly, the relative accumulation of transcripts corresponding to various 
alternative oxidase (AOX) and rotenone-insensitive $\mathrm{NAD}(\mathrm{P}) \mathrm{H}$ dehydrogenase (NDs) mRNAs in misf2 was generally higher than in wild type plants (Figure 8b). Similarly, immunoblot assays indicated that the steady-state levels of AOX1 or AOX2 proteins was higher in misf2 compared to the wild type (Figure 8a and S4).

\section{Discussion}

3.1. The MISF2 gene encodes a mitochondrion localized PPR protein, which is playing essential roles during early embryo-development in Arabidopsis plants

Mitochondria play key roles in energy metabolism and are thus vital organelle for plant life. During the evolution, the mitochondrial genomes of land plants have undergone increased plasticity, showing substantial variations in genome size and structures, and gene expression patterns between species (reviewed by e.g., [1]). Angiosperm mtDNAs are the largest and least gene-dense genomes among eukaryotes [2]. mRNA production and expression in land plant mitochondria involve extensive processing steps, which include endonucleolytic cleavages, 5' and 3' mRNA trimming, extensive sequence editing and, relevantly to our study, the removal of intron (mostly group II-type) sequences that interrupt the coding regions of many mitochondrial genes (reviewed by e.g., [6]). These essential activities may serve as key control points of plant mitochondrial gene expression and are facilitated by numerous RNA binding cofactors [6, 8].

In this study, we assigned a role to an Arabidopsis PPR protein, namely the MISF2 encoded by the AT3G22670 gene-locus in mt-RNA metabolism (i.e., nad2 maturation) and respiratoty complex I assembly. MISF2, like its maize orthologue, EMP10 [41], is a P-type PPR protein comprising 10 PPR motifs that is localized in mitochondria. As for EMP10 in maize, downregulation of MISF2 expression results in premature arrest of Arabidopsis embryo development at the late torpedo stage (Figure 1B). The loci of MISF and RDM1 genes in Arabidopsis are partially overlapping. It was therefore impotrant to confirm that the developmental defect phenotypes and alterd mt-RNA metabolism we see in misf2 mutants result directly from the downregulation of MISF2 expression. To this aim, we analyzed the growth phenotypes and organellar RNA and protein profiles associated with a functionaly complemented misf2 line, and further showed that MISF2 is directly required for nad2 RNA maturation and resiratory complex I biogenesis and respiratory functions.

\subsection{MISF2 is required for the splicing of nad2 intron 1}

Most mitochondrial introns in angiosperms are classified as group II-type [12]. Model introns belonging to this class are large catalytic RNAs that are characterized by a conserved secondary structure consisting of six double-helical domains (named domains I to VI), radiating from a central hub $[77,78]$. The excision of group II introns in vivo in bacteria and in the organelles of eukaryotic cells requires the action of various RNA binding protein cofactors. In canonical group II introns, these at least include maturase proteins that are most often encoded by the introns themselves [79]. In plant mitochondria, many additional proteinaceous splicing factors are required, which either derive from an ancient group of maturases [14], or from various other RNA binding cofactors that were recruited along evolution to facilitate mitochondrial intron splicing $[13,80]$.

The PPR protein family is the largest RNA binding protein family known in plants, with about 400 to 600 members targeted to mitochondria and plastids [81]. PPR proteins bind their RNA substrates in a sequence specific manner, and were shown to play pivotal roles in various aspects of posttranscriptional RNA processing, including the excision of group II introns in land plant organelles [6, 9, 27, 40]. Here, we analyzed the molecular functions of the Arabidopsis MISF2 protein by characterizing loss-of-function mutants. As no homozygous mutant individuals could be identified among mature seeds of selffertilized heterozygous misf2 progenies, we used embryo rescue approaches [52, 56] to generate homozygous mutant plant material, which allowed us to analyze the role of MISF2 in mitochondrial RNA metabolism. 
Analysis of mitochondrial RNA profiles in wild type and misf2 plants showed a large reduction in the accumulation of mature nad2 mRNA in both mutant lines (Figure 3 ). The RT-qPCR analyses further revealed a strong reduction in the splicing efficiency of nad2 intron 1 in misf2 plants (Figure 4). The most probable RNA-binding site for MISF2 protein (i.e., GGUGAGGCGU) resides within the domain I of nad2 intron 1 (Figure 5), which also corresponds to the genetic and biochemical RNA target of MISF2 (Figures 3, 4 and 6). In model group II introns, maturases were shown to bind with great affinity and specificity to their cognate intron-RNAs, in particular to regions of domain I and around the maturase coding sequences within domain IV [82]. It will therefore be interesting to investigate whether sequence changes within plant nad2 intron 1 was accompanied by the recruitment of the PPR MISF2 factor to facilitate its splicing, possibly to stabilize (or nucleate) nad 2 intron 1 folding into a catalytically active structure.

Our data provide strong evidence that MISF2 is specifically required for nad2 intron 1 splicing and that this RNA processing step is essential for early embryogenesis in Arabidopsis.

\subsection{Embryo development and complex I biogenesis and activity}

The electron transport chain is made of four major multi-subunit protein complexes, denoted as CI to CIV. Plants also possess several enzymes corresponding to non-phosphorylating bypasses of the electron transport chain (ETC), namely the alternative oxidase $(\mathrm{AOX})$ and several rotenone-insensitive NAD $(\mathrm{P}) \mathrm{H}$ dehydrogenases (NDs) $[64,83-86]$. The biogenesis of respiratory $\mathrm{CI}$ in angiosperms involves the incorporation of $\sim 50$ different subunits encoded by both mitochondrial (i.e., nad1, nad2, nad3, nad4, nad4l, nad5, nad6, nad7 and nad9) and nuclear gene loci [87]. These are incorporated into two main different CI domains, consisting in a membrane domain and the matrix (or peripheral) arm $[67,76,88$, 89].

Nad2 is a pivotal subunit of $\mathrm{CI}$, that is suggested to be incorporated very early during the assembly of the membrane arm $[67,88,90,91]$. The early steps of CI biogenesis involve the production of an $\sim 85 \mathrm{kDa}$ assembly intermediate of the membrane arm, which contains various gamma-type carbonic anhydrase subunits. Subsequently, Nad2 and few other subunits are incorporated to form a $\sim 200 \mathrm{kDa}$ membrane-bound CI assembly intermediate [67]. It is therefore anticipated that a strong reduction in Nad2 would interfere with the assembly of the CI membrane arm, and hence with the biogenesis of the $\sim 1.0$ MDa holo-CI. Consequently, BN-PAGE analysis of wild type and mutant plants revealed a major reduction in CI abundance in both misf2 mutant lines (Figure 7). Immunoblot analysis with anti-CA2 antibodies further revealed the existence of various CI intermediates in misf2 mutants, among which a major particle of about $85 \mathrm{kDa}$, which was also observed in the abo5 mutant that is impaired in nad2 expression [69]. The CI particles of higher mass (i.e., $230 \mathrm{kDa}$ and $610 \mathrm{kDa}$ ) detected in the mutants may correspond to Nad2deprived assembly intermediates that are less stable than the $\sim 85 \mathrm{kDa}$ particles [67].

It has been demonstrated that the severity of CI production defects correlates with the gravity of the phenotypes displayed by corresponding plant mutants $[53,72,76]$. Severe CI mutants are impaired in the storage of essential nutrients, but not in the mobilization of the stored reserves [53]. Accordingly, mutants affected in ๑-oxidation, a metabolic process by which fatty acids are broken down by various tissues to produce energy, contain embryos that are typically arrested at earlier developmental stages compared with CI mutants [92]. Embryo maturation is often incomplete in various CI mutants, leading to the production of seeds with reduced reserves and germination capacity. One can anticipate that altered respiration interferes with numerous essential metabolic activities, resulting in altered embryo development.

In our study, we noticed that a severe defect in the production of the Nad2 subunit of CI results in impaired embryogenesis and a loss of germination capacity of mutant Arabidopsis seeds. However, most characterized plant CI mutants are generally able to germinate under standard culture conditions (see e.g., [17, 71, 72, 93-95]. The inability of misf2 
mutants to germinate under normal conditions is expected to result from an early arrest of the development of mutant embryos, placing misf 2 mutants among the most severe CI mutants reported so far. We currently do not know the role that the embryo-rescue medium plays in improving the seed germination of misf2 mutants. It may be due to the presence of certain important chemicals in the rescue medium, or simply to a weakening of the seed coat by the high sugar concentration of the medium. Once germination has been induced, we could observe that misf2 mutants often show growth phenotypes like other Arabidopsis CI mutants (Figure 1b-iv). It was previously suggested that once photosynthesis is established, growth is to a lesser extent dependent on the application of external vitamins and/or sugars [53]. Subsequently, rescued misf2 mutants can slowly proceed with their vegetative growth phase, but remain unable to complete their life cycle, flower and produce viable seeds.

\section{Materials and Methods}

\subsection{Plant material and growth conditions}

Arabidopsis thaliana of the Columbia (Col-0) accession was used in all experiments. The wild type (Col-0 line) and the SALK-067654 (misf2.1) and SALK-066141 (misf2.2) mutants were obtained from the Arabidopsis Biological Resource Center (ABRC). Prior to germination, seeds obtained from wild type and mutant lines were surface-sterilized with $\mathrm{Cl}_{2}$ gas, generated by the addition of $1 \mathrm{ml} \mathrm{HCl}$ per $50 \mathrm{~mL}$ of bleach (sodium hypochlorite $4.7 \%$ ), for 4 hours at room-temperature (RT). The seeds were then sown on MS-agar plates containing $1 \%(\mathrm{w} / \mathrm{v})$ sucrose or rescued by a method described in detail below. For synchronized germinations, the seeds were kept in the dark for 5 days at $4^{\circ} \mathrm{C}$ and then grown under long day condition (LD, 16:8-hour) in a growth chamber (Percival Scientific, Perry, IA, USA) at $22^{\circ} \mathrm{C}$ and under light intensity of $300 \mu \mathrm{E} \mathrm{m}^{-2} \mathrm{~s}^{-1}$. PCR was used for genotyping the plants using specific oligonucleotides listed in Table S1. Sequencing of specific PCR products was used to check the T-DNA insertion site in both mutant lines.

\subsection{GFP localization assay}

The DNA region encoding the first 203 amino acids of MISF2 was PCR amplified with specific oligonucleotides (i.e., misf2-B1 and misf2-B2; Table S1C). The 609 nts PCR DNA fragment was cloned into pDONR207 vector using the Gateway BP clonase enzyme mix and verified by Sanger sequencing. The entry clone was then transferred into pGWB5 vector by Gateway LR reaction to create a GFP translational fusion between the MISF2 Nterminus sequence and the GFP coding sequence. The vector was transformed into Agrobacterium tumefaciens (strain C58C51) and used to transform Arabidopsis plant cells. Transgenic cells were selected on hygromycin, and GFP fluorescence was visualized by confocal microscopy Leica TCS SP8. To visualize mitochondria in vivo, plant cells were incubated with $1 \mu \mathrm{M}$ MitoTracker ${ }^{\circledR}$ Red (Thermofisher) for $10 \mathrm{~min}$ at room temperature prior to observation under confocal microscopy.

\subsection{Embryo-rescue and establishment of homozygous misf2 mutants}

Siliques from wild type and heterozygous misf2 plants were surfaced sterilized with $6 \%$ bleach solution for $10 \mathrm{~min}$ at RT. The seeds were then soaked in a $70 \%$ ethanol solution for $10 \mathrm{~min}$ at RT, washed briefly with sterile DDW, and opened in a sterile hood. Green and white seeds obtained from siliques of heterozygous misf2 plants 10 days after selffertilization were sown on MS-agar plates supplemented with 1\% (w/v) sucrose and 10 mg myoinositol, $100 \mu \mathrm{g}$ thiamine, $100 \mu \mathrm{g}$ pyridoxine and $100 \mu \mathrm{g}$ nicotinic acid. For DNA and RNA analysis we used Arabidopsis wild type and misf2 plantlets at stage R6 (i.e., 6 to 8 leaves) [57]. To obtain larger quantities of plant material, plantlets at stage R6 were grown on MS-agar plates and then transferred to MS-based liquid medium supplemented with 1\% (w/v) sucrose and 10 mg myoinositol $100 \mu \mathrm{g}$ Thiamine, $100 \mu \mathrm{g}$ Pyridoxine, 100 
$\mu \mathrm{g}$ nicotinic acid and incubated at $22^{\circ} \mathrm{C}$ and under a light intensity of $300 \mu \mathrm{E} \mathrm{m}-2 \mathrm{~s}^{-1}$ with moderate (50 100 RPM) shaking.

\subsection{Microscopic analyses of Arabidopsis wild type and mutant plants}

Analysis of whole plant morphology, roots, leaves, siliques and seeds of wild type and mutant lines were examined under Stereoscopic (dissecting) microscope or light microscope at the Bio-Imaging unit of the Institute of Life Sciences (The Hebrew University of Jerusalem). Seeds were incubated with Hoyer solution for 30 minutes and the cleared samples were analyzed by differential interference contrast (Nomarski) microscopy.

\subsection{RNA extraction and analysis}

RNA extraction and analysis was performed essentially as previously described [17, 19, 96-98]. Total RNA was prepared from $200 \mathrm{mg}$ seedlings grown on MS-agar plates supplemented with $1 \%$ sucrose, using the RNAzol RT reagent (Sigma-Aldrich). The RNA was then treated with RNase-free DNase I prior to its use in the assays. RT-qPCR was performed with specific oligonucleotides designed to exon-exon (mRNAs) regions corresponding to mitochondrial genes and intron-exon regions (pre-mRNAs) within each of the 23 group II introns in Arabidopsis thaliana (Tables S1). cDNA was synthesized by reverse transcription with the Superscript III reverse transcriptase (Invitrogen), using 1 - 2 $\mu \mathrm{g}$ of total RNA and $250 \mathrm{ng}$ of a mixture of random hexanucleotides (Promega) and incubated for $50 \mathrm{~min}$ at $50^{\circ} \mathrm{C}$. Reactions were stopped by $15 \mathrm{~min}$ incubation at $70^{\circ} \mathrm{C}$ and the RT samples served directly for real-time PCR on a LightCycler 480 (Roche) using $2.5 \mu \mathrm{L}$ of LightCycler 480 SYBR Green I Master mix and $2.5 \mu \mathrm{M}$ of primers in a final volume of 5 $\mu \mathrm{L}$. Reactions were performed in triplicate in the following conditions: pre-heating at $95^{\circ} \mathrm{C}$ for $10 \mathrm{~min}$, followed by 40 cycles of $10 \mathrm{sec}$ at $95^{\circ} \mathrm{C}, 10 \mathrm{sec}$ at $58^{\circ} \mathrm{C}$ and $10 \mathrm{sec}$ at $72^{\circ} \mathrm{C}$. The nucleus-encoded 18S rRNA (At3g41768) and the mitochondrial 26S ribosomal rRNA subunit (ArthMr001) were used as reference genes.

\subsection{Crude mitochondria preparations}

Crude mitochondria extracts prepared essentially as described previously [74]. To this end, $200 \mathrm{mg}$ of plantlets grown in liquid culture were harvested and homogenized in $2 \mathrm{ml}$ of $75 \mathrm{mM}$ MOPS-KOH, pH 7.6, $0.6 \mathrm{M}$ sucrose, $4 \mathrm{mM}$ EDTA, $0.2 \%$ polyvinylpyrrolidone- $40,8 \mathrm{mM}$ L-cysteine, $0.2 \%$ bovine serum albumin and protease inhibitor cocktail 'complete Mini' from Roche Diagnostics GmbH (Mannheim, Germany). The lysate was filtrated through one layer of Miracloth and centrifuged at $1,300 \mathrm{~g}$ for $4 \mathrm{~min}$ at $4^{\circ} \mathrm{C}$ (to remove cell debris). The supernatant was then centrifuged at 22,000 $\mathrm{g}$ for $10 \mathrm{~min}$ at $4^{\circ} \mathrm{C}$. The resulting pellet containing thylakoid and mitochondrial membranes was washed twice with $1 \mathrm{ml}$ of wash buffer $37.5 \mathrm{mM}$ MOPS-KOH, $0.3 \mathrm{M}$ sucrose and 2mM EDTA, pH 7.6 prior to use.

\subsection{Blue native PAGE analysis of respiratory complexes}

Blue native (BN)-PAGE of crude organellar membranous fractions was performed according to the method described in Pineau, Layoune, Danon and De Paepe [74]. An aliquot equivalent to $40 \mathrm{mg}$ of crude Arabidopsis mitochondria extracts was solubilized with 5\% (w/v) digitonin in BN-solubilization buffer (30 mM HEPES, pH 7.4, 150 mM potassium acetate, $10 \%[\mathrm{v} / \mathrm{v}]$ glycerol) and then incubated on ice for $30 \mathrm{~min}$. The samples were centrifuged $8 \mathrm{~min}$ at 20,000 xg to pellet non-solubilized material and $0.2 \%$ [v/v] of Serva Blue $G$ was added to the supernatant. The samples were then loaded onto a native 4 to $16 \%$ linear gradient gel. For 'non-denaturing-PAGE' immunoblotting, the gel was transferred to a PVDF membrane (Bio-Rad) in Cathode buffer (50 mM Tricine and $15 \mathrm{mM}$ Bis-Tris- $\mathrm{HCl}, \mathrm{pH} 7.0$ ) for $16 \mathrm{~h}$ at $4^{\circ} \mathrm{C}$ at constant current of $40 \mathrm{~mA}$. The blots where then incubated with antibodies against mitochondrial proteins (Table S2) and hybridization 
signals were identified by chemiluminescence assay after incubation with an appropriate horseradish peroxidase (HRP)-conjugated secondary antibody.

\subsection{RNA co-immunoprecipitation assays}

Immunoprecipitation of MISF2-3HA were performed using the $\mu$ MACS HA-Tagged Protein Isolation Kit (Miltenyi Biotec) following a procedure previously described in Wang et al, 2020 [80].

\section{Conclusions}

Angiosperms encode numerous PPR proteins that are predominantly localized in plastids and mitochondria, and which carry essential roles in organellar RNA metabolism. These include the EMP10 protein, which regulates the maturation of nad2 in maize mitochondria [41]. Analysis of protein and RNA profiles of mutants affected in the Arabidopsis orthologous gene, designated MITOCHONDRIAL SPLICING FACTOR 2 (MISF2, encoded by At3g22670 gene), indicates that MISF2 also functions specifically in the excision of the first intron of nad2. Plant mutants affected in MISF2 accumulate high levels of nad2 pre-RNA in vivo and show a notable reduction in nad 2 intron 1 splicing. The splicing defect found in misf2 (or emp10) is associated with altered CI biogenesis and arrested embryonic development. Together, these data show that the molecular functions are conserved between the Arabidopsis MISF2 protein and its related EMP10 homolog in maize [41], which suggests that the common PPR ancestor of MISF2 and EMP10 has been recruited to act in nad2 intron 1 splicing before the divergence of monocot and dicot plant species [42]. Our results provide important insights into the roles of nuclear-encoded PPR factors in mitochondria gene expression and the biogenesis of the respiratory system during early plant life.

Supplementary Materials: The following supporting information can be downloaded at: www.mdpi.com/xxx/s1, Figure S1: The topology and structure of MISF2 protein.; Figure S2: MISF2 gene expression pattern during Arabidopsis development.; Figure S3: Nucleotide sequence of the MISF2 gene and precise location of T-DNA insertion site.; Figure S4: Steady-state level analysis of various mitochondrial proteins in wild type, misf2.2 and functionally complemented misf2.2 plants.; Table S1: List of oligonucleotides used in this study.; Table S2: List of antibodies used in this study.

Author Contributions: methodology, T-T.N. C.B., S.S., M.Z., M.Q., R.M., H.Z., H.M. and O.O.B.; formal analysis, T-T.N. C.B., S.S., M.Z., M.Q., R.M., H.Z., H.M. and O.O.B.; data curation, T-T.N., H.M., R.M. and O.O.B.; writing - original draft preparation, H.M. and O.O.B.; writing - review and editing, H.M., T-T.N., R.M. and O.O.B.; supervision, H.M. and O.O.B.; funding acquisition, H.M. and O.O.B. All authors have read and agreed to the published version of the manuscript.

Funding: This work was supported by grants to O.O.B from the 'Israeli Science Foundation' ISF grants no. 1834/20 and 3254-2020 and by grants to H. M. from the French National Research Agency no. ANR-16-CE11- 0024-01. The IJPB benefits from the support of Saclay Plant Sciences-SPS (ANR17-EUR-0007). This work has benefited from the support of IJPB's Plant Observatory technological platforms.

Informed Consent Statement: Not applicable.

Acknowledgments: We thank Prof. Dr. Eduardo Zabaleta (UNMDP) for providing us with antiCA2 antibodies. The authors also wish to thank Prof. Ariel Chipman (HUJI) for his help with microscopy analyses. We also wish to thank Omer Ben-Dor (HUJI) for his help with misf2 mutants screening.

Conflicts of Interest: The authors declare no conflict of interest.

\section{References}

1. Best, C.; Mizrahi, R.; Ostersetzer-Biran, O., Why so complex? the intricacy of genome structure and gene expression, associated with angiosperm mitochondria, may relate to the regulation of embryo quiescence or dormancy-intrinsic blocks to early plant life. Plants 2020, 9, (5), 598. 
2. Gualberto, J. M.; Newton, K. J., Plant mitochondrial genomes: Dynamics and mechanisms of mutation. Annu Rev Plant Biol 2017, 68, 225-252.

3. Woodson, J. D.; Chory, J., Coordination of gene expression between organellar and nuclear genomes. Nat Rev Genet 2008, 9, (5), 383-395.

4. Kleine, T.; Leister, D., Retrograde signaling: Organelles go networking. BBA 2016, 1857, (8), 1313-25.

5. Fuchs, P.; Rugen, N.; Carrie, C.; Elsasser, M.; Finkemeier, I.; Giese, J.; Hildebrandt, T. M.; Kuhn, K.; Maurino, V. G.; Ruberti, C.; Schallenberg-Rudinger, M.; Steinbeck, J.; Braun, H. P.; Eubel, H.; Meyer, E. H.; Muller-Schussele, S. J.; Schwarzlander, M., Single organelle function and organization as estimated from Arabidopsis mitochondrial proteomics. Plant J 2020, 101, (2), 420-441.

6. Zmudjak, M.; Ostersetzer-Biran, O., RNA metabolism and transcript regulation. Chichester John Wiley \& Sons, Ltd: 2017; Vol. 50, p 143-184.

7. Colas des Francs-Small, C.; Small, I., Surrogate mutants for studying mitochondrially encoded functions. Biochimie 2014, 100, (0), 234-242.

8. Hammani, K.; Giege, P., RNA metabolism in plant mitochondria. Trends Plant Sci 2014, 19, (6), 380-9.

9. Small, I. D.; Schallenberg-Rudinger, M.; Takenaka, M.; Mireau, H.; Ostersetzer-Biran, O., Plant organellar RNA editing: what 30 years of research has revealed. Plant J 2020, 101, (5), 1040-1056.

10. Michel, F.; Lang, B. F., Mitochondrial class II introns encode proteins related to the reverse transcriptases of retroviruses. Nature 1985, 316, (6029), 641-3.

11. Sharp, P. A., On the origin of RNA splicing and introns. Cell 1985, 42, (2), 397-400.

12. Bonen, L., Cis- and trans-splicing of group II introns in plant mitochondria. Mitochondrion 2008, 8, (1), $26-34$.

13. Brown, G. G.; Colas des Francs-Small, C.; Ostersetzer-Biran, O., Group II intron splicing factors in plant mitochondria. Front Plant Sci 2014, 5, (35), 1-13.

14. Schmitz-Linneweber, C.; Lampe, M.-K.; Sultan, L. D.; Ostersetzer-Biran, O., Organellar maturases: A window into the evolution of the spliceosome. BBA - Bioenergetics 2015, 1847, ((9)), 798-808.

15. Mohr, G.; Lambowitz, A. M., Putative proteins related to group II intron reverse transcriptase/maturases are encoded by nuclear genes in higher plants. Nucleic Acids Res 2003, 31, (2), 647-652.

16. Köhler, D.; Schmidt-Gattung, S.; Binder, S., The DEAD-box protein PMH2 is required for efficient group II intron splicing in mitochondria of Arabidopsis thaliana. Plant Mol Biol 2010, 72, (4), 459-467.

17. Zmudjak, M.; Shevtsov, S.; Sultan, L. D.; Keren, I.; Ostersetzer-Biran, O., Analysis of the roles of the Arabidopsis nMAT2 and $\mathrm{PMH} 2$ proteins provided with new insights into the regulation of group II intron splicing in land-plant mitochondria. Int $J$ Mol Sci 2017, 18, (11), 1-25.

18. He, J.; Duan, Y.; Hua, D.; Fan, G.; Wang, L.; Liu, Y.; Chen, Z.; Han, L.; Qu, L.-J.; Gong, Z., DEXH box RNA helicase-mediated mitochondrial reactive oxygen species production in Arabidopsis mediates crosstalk between abscisic acid and auxin signaling. Plant Cell 2012, 24, (5), 1815-1833.

19. Colas des Francs-Small, C.; Kroeger, T.; Zmudjak, M.; Ostersetzer-Biran, O.; Rahimi, N.; Small, I.; Barkan, A., A PORR domain protein required for rpl2 and ccmFc intron splicing and for the biogenesis of c-type cytochromes in Arabidopsis mitochondria. Plant J 2012, 69, (6), 996-1005.

20. Peeters, N.; Small, I., Dual targeting to mitochondria and chloroplasts. BBA 2001, 1541, 54-63.

21. Chen, L.; Li, Y.-x.; Li, C.; Shi, Y.; Song, Y.; Zhang, D.; Li, Y.; Wang, T., Genome-wide analysis of the pentatricopeptide repeat gene family in different maize genomes and its important role in kernel development. BMC Plant Biol 2018, 18, (1), 366.

22. Lurin, C.; Andres, C.; Aubourg, S.; Bellaoui, M.; Bitton, F.; Bruyere, C.; Caboche, M.; Debast, C.; Gualberto, J.; Hoffmann, B.; Lecharny, A.; Le Ret, M.; Martin-Magniette, M.-L.; Mireau, H.; Peeters, N.; Renou, J.-P.; Szurek, B.; Taconnat, L.; Small, I., Genome-wide analysis of Arabidopsis pentatricopeptide repeat proteins reveals their essential role in organelle biogenesis. Plant Cell 2004, 16, (8), 2089-2103.

23. Schmitz-Linneweber, C.; Small, I., Pentatricopeptide repeat proteins: a socket set for organelle gene expression. Trends Plant Sci 2008, 13, (12), 663-670.

24. Coquille, S.; Filipovska, A.; Chia, T.; Rajappa, L.; Lingford, J. P.; Razif, M. F. M.; Thore, S.; Rackham, O., An artificial PPR scaffold for programmable RNA recognition. Nat Comm 2014, 5, 5729.

25. Gully, B. S.; Cowieson, N.; Stanley, W. A.; Shearston, K.; Small, I. D.; Barkan, A.; Bond, C. S., The solution structure of the pentatricopeptide repeat protein PPR10 upon binding atpH RNA. Nucleic Acids Res 2015, 43, (3), 1918-26.

26. Binder, S.; Hölzle, A.; Jonietz, C., RNA processing and RNA stability in plant mitochondria. In Plant mitochondria, Springer: 2011; pp 107-130.

27. Barkan, A.; Small, I., Pentatricopeptide Repeat Proteins in Plants. Annu Rev Plant Biol 2014, 65, (1), 415-442.

28. Shikanai, T.; Fujii, S., Function of PPR proteins in plastid gene expression. RNA Biol 2013, 10, (9), 1446-1456.

29. Geddy, R.; Brown, G. G., Genes encoding pentatricopeptide repeat (PPR) proteins are not conserved in location in plant genomes and may be subject to diversifying selection. BMC Genomics 2007, 8, 130-130.

30. Dahan, J.; Mireau, H., The Rf and Rf-like PPR in higher plants, a fast-evolving subclass of PPR genes. RNA biol 2013, 10, (9), 1469-76.

31. Zhao, N.; Wang, Y.; Hua, J., Genomewide identification of PPR gene family and prediction analysis on restorer gene in Gossypium. J Genet 2018, 97, (5), 1083-1095. 
32. Barkan, A.; Rojas, M.; Fujii, S.; Yap, A.; Chong, Y.; Bond, C.; Small, I., A combinatorial amino acid code for RNA recognition by pentatricopeptide repeat proteins. PLoS Genet 2012, 8, e1002910.

33. Shen, C.; Zhang, D.; Yan, J.; Zhang, Q.; Hong, S.; Yang, Y.; Yao, Y.; Yin, P.; Zou, T., Delineation of pentatricopeptide repeat codes for target RNA prediction. Nucleic Acids Res 2019, 47, (7), 3728-3738.

34. Yin, P.; Li, Q.; Yan, C.; Liu, Y.; Liu, J.; Yu, F.; Wang, Z.; Long, J.; He, J.; Wang, H.-W.; Wang, J.; Zhu, J.-K.; Shi, Y.; Yan, N., Structural basis for the modular recognition of single-stranded RNA by PPR proteins. Nature 2013, $504,168$.

35. Ke, J.; Chen, R. Z.; Ban, T.; Zhou, X. E.; Gu, X.; Tan, M. H.; Chen, C.; Kang, Y.; Brunzelle, J. S.; Zhu, J. K.; Melcher, K.; Xu, H. E., Structural basis for RNA recognition by a dimeric PPR-protein complex. Nat Struc Mol Biol 2013, 20, (12), $1377-82$.

36. Gully, B. S.; Shah, K. R.; Lee, M.; Shearston, K.; Smith, N. M.; Sadowska, A.; Blythe, A. J.; Bernath-Levin, K.; Stanley, W. A.; Small, I. D.; Bond, C. S., The design and structural characterization of a synthetic pentatricopeptide repeat protein. Acta Crystal 2015, 71, (Pt 2), 196-208.

37. Shen, C.; Zhang, D.; Guan, Z.; Liu, Y.; Yang, Z.; Yang, Y.; Wang, X.; Wang, Q.; Zhang, Q.; Fan, S.; Zou, T.; Yin, P., Structural basis for specific single-stranded RNA recognition by designer pentatricopeptide repeat proteins. Nat Comm 2016, 7, 11285.

38. Brehme, N.; Zehrmann, A.; Verbitskiy, D.; Hartel, B.; Takenaka, M., Mitochondrial RNA editing PPR proteins can tolerate protein tags at $\mathrm{E}$ as well as at DYW domain termini. Front Plant Sci 2014, 5 (127), 1-4.

39. Takenaka, M.; Jörg, A.; Burger, M.; Haag, S., RNA editing mutants as surrogates for mitochondrial SNP mutants. Plant Physiol Biochem 2019, 135, 310-321.

40. Rovira, A. G.; Smith, A. G., PPR proteins - orchestrators of organelle RNA metabolism. Physiol Plant 2019, 166, (1), 451-459.

41. Cai, M.; Li, S.; Sun, F.; Sun, Q.; Zhao, H.; Ren, X.; Zhao, Y.; Tan, B. C.; Zhang, Z.; Qiu, F., Emp10 encodes a mitochondrial PPR protein that affects the cis-splicing of nad2 intron 1 and seed development in maize. Plant J 2017, 91, (1), $132-144$.

42. Wolfe, K. H.; Gouy, M.; Yang, Y. W.; Sharp, P. M.; Li, W. H., Date of the monocot-dicot divergence estimated from chloroplast DNA sequence data. P Natl Acad Sci USA 1989, 86, (16), 6201-6205.

43. Gutmann, B.; Royan, S.; Schallenberg-Rüdinger, M.; Lenz, H.; Castleden, I. R.; McDowell, R.; Vacher, M. A.; Tonti-Filippini, J.; Bond, C. S.; Knoop, V.; Small, I. D., The Expansion and Diversification of Pentatricopeptide Repeat RNA-Editing Factors in Plants. Mol Plant 2020, 13, (2), 215-230.

44. Letunic, I.; Doerks, T.; Bork, P., SMART 7: recent updates to the protein domain annotation resource. Nucleic Acids Res 2012, 40, D302-D305.

45. Marchler-Bauer, A.; Anderson, J. B.; DeWeese-Scott, C.; Fedorova, N. D.; Geer, L. Y.; He, S.; Hurwitz, D. I.; Jackson, J. D.; Jacobs, A. R.; Lanczycki, C. J.; Liebert, C. A.; Liu, C.; Madej, T.; Marchler, G. H.; Mazumder, R.; Nikolskaya, A. N.; Panchenko, A. R.; Rao, B. S.; Shoemaker, B. A.; Simonyan, V.; Song, J. S.; Thiessen, P. A.; Vasudevan, S.; Wang, Y.; Yamashita, R. A.; Yin, J. J.; Bryant, S. H., CDD: a curated Entrez database of conserved domain alignments. Nucleic Acids Res 2003, 31, (1), 383-387.

46. Wu, C. H.; Apweiler, R.; Bairoch, A.; Natale, D. A.; Barker, W. C.; Boeckmann, B.; Ferro, S.; Gasteiger, E.; Huang, H.; Lopez, R.; Magrane, M.; Martin, M. J.; Mazumder, R.; O'Donovan, C.; Redaschi, N.; Suzek, B., The Universal Protein Resource (UniProt): an expanding universe of protein information. Nucleic Acids Res 2006, 34, (Database issue), D187-91.

47. Hooper, C. M.; Castleden, I. R.; Tanz, S. K.; Aryamanesh, N.; Millar, A. H., SUBA4: the interactive data analysis centre for Arabidopsis subcellular protein locations. Nucleic Acids Res 2017, 45, (D1), D1064-D1074.

48. Jumper, J.; Evans, R.; Pritzel, A.; Green, T.; Figurnov, M.; Ronneberger, O.; Tunyasuvunakool, K.; Bates, R.; Žídek, A.; Potapenko, A.; Bridgland, A.; Meyer, C.; Kohl, S. A. A.; Ballard, A. J.; Cowie, A.; Romera-Paredes, B.; Nikolov, S.; Jain, R.; Adler, J.; Back, T.; Petersen, S.; Reiman, D.; Clancy, E.; Zielinski, M.; Steinegger, M.; Pacholska, M.; Berghammer, T.; Bodenstein, S.; Silver, D.; Vinyals, O.; Senior, A. W.; Kavukcuoglu, K.; Kohli, P.; Hassabis, D., Highly accurate protein structure prediction with AlphaFold. Nature 2021, 596, (7873), 583-589.

49. Hruz, T.; Laule, O.; Szabo, G.; Wessendorp, F.; Bleuler, S.; Oertle, L.; Widmayer, P.; Gruissem, W.; Zimmermann, P., Genevestigator V3: a reference expression database for the meta-analysis of transcriptomes. Adv Bioinform 2008, 2008, 420747-420751.

50. Meinke, D. W., Genome-wide identification of EMBRYO-DEFECTIVE (EMB) genes required for growth and development in Arabidopsis. New Phytol 2020, 226, (2), 306-325.

51. Franzmann, L.; Patton, D. A.; Meinke, D. W., In vitro morphogenesis of arrested embryos from lethal mutants of Arabidopsis thaliana. Theor Appl Genet 1989, 77, (5), 609-616.

52. Dahan, J.; Tcherkez, G.; Macherel, D.; Benamar, A.; Belcram, K.; Quadrado, M.; Arnal, N.; Mireau, H., Disruption of the CYTOCHROME C OXIDASE DEFICIENT1 gene leads to cytochrome c oxidase depletion and reorchestrated respiratory metabolism in Arabidopsis. Plant Physiol 2014, 166, (4), 1788-802.

53. Kuhn, K.; Obata, T.; Feher, K.; Bock, R.; Fernie, A. R.; Meyer, E. H., Complete mitochondrial complex I deficiency induces an up-regulation of respiratory fluxes that is abolished by traces of functional complex I. Plant Physiol 2015, 168, (4), 1537-49.

54. Cordoba, J. P.; Marchetti, F.; Soto, D.; Martin, M. V.; Pagnussat, G. C.; Zabaleta, E., The CA domain of the respiratory complex I is required for normal embryogenesis in Arabidopsis thaliana. J Exp Bot 2016, 67, (5), 1589-603.

55. Fromm, S.; Going, J.; Lorenz, C.; Peterhansel, C.; Braun, H. P., Depletion of the "gamma-type carbonic anhydrase-like" subunits of complex I affects central mitochondrial metabolism in Arabidopsis thaliana. BBA 2016, 1857, (1), 60-71.

56. Shevtsov-Tal, S.; Best, C.; Matan, R.; Chandran, S. A.; Brown, G. G.; Ostersetzer-Biran, O., nMAT3 is an essential maturase splicing factor required for holo-complex I biogenesis and embryo development in Arabidopsis thaliana plants. Plant J 2021, $106,(4), 1128-1147$. 
57. Boyes, D. C.; Zayed, A. M.; Ascenzi, R.; McCaskill, A. J.; Hoffman, N. E.; Davis, K. R.; Görlach, J., Growth Stage-Based Phenotypic Analysis of Arabidopsis. A Model for High Throughput Functional Genomics in Plants. Plant Cell 2001, 13, (7), 1499-1510.

58. Sasaki, T.; Lorković, Z. J.; Liang, S.-C.; Matzke, A. J. M.; Matzke, M., The Ability to form homodimers is essential for RDM1 to function in RNA-directed dna methylation. PLoS ONE 2014, 9, (2), e88190.

59. Yagi, Y.; Hayashi, S.; Kobayashi, K.; Hirayama, T.; Nakamura, T., Elucidation of the RNA recognition code for pentatricopeptide repeat proteins involved in organelle RNA editing in plants. PLoS ONE 2013, 8, e57286.

60. Yagi, Y.; Nakamura, T.; Small, I., The potential for manipulating RNA with pentatricopeptide repeat proteins. Plant J 2014, 78, (5), 772-782.

61. Takenaka, M.; Zehrmann, A.; Brennicke, A.; Graichen, K., Improved Computational target site prediction for pentatricopeptide repeat RNA editing factors. PLoS ONE 2013, 8, (6), e65343.

62. Cheng, S.; Gutmann, B.; Zhong, X.; Ye, Y.; Fisher, M. F.; Bai, F.; Castleden, I.; Song, Y.; Song, B.; Huang, J.; Liu, X.; Xu, X.; Lim, B. L.; Bond, C. S.; Yiu, S. M.; Small, I., Redefining the structural motifs that determine RNA binding and RNA editing by pentatricopeptide repeat proteins in land plants. Plant J 2016, 85, (4), 532-47.

63. Klodmann, J.; Senkler, M.; Rode, C.; Braun, H.-P., Defining the "protein complex proteome" of plant mitochondria. Plant Physiol 2011, 157, (2), 587-98.

64. Millar, A. H.; Whelan, J.; Soole, K. L.; Day, D. A., Organization and regulation of mitochondrial respiration in plants. Ann Rev Plant Biol 2011, 62, (1), 79-104.

65. Zhao, P.; Wang, F.; Li, N.; Shi, D.-Q.; Yang, W.-C., Pentatricopeptide repeat protein MID1 modulates nad2 intron 1 splicing and Arabidopsis development. Scientific Reports 2020, 10, (1), 2008.

66. Marchetti, F.; Cainzos, M.; Shevtsov, S.; Cordoba, J. P.; Sultan, L. D.; Brennicke, A.; Takenaka, M.; Pagnussat, G.; OstersetzerBiran, O.; Zabaleta, E., Mitochondrial Pentatricopeptide repeat protein, EMB2794, plays a pivotal role in nadh dehydrogenase subunit nad2 mRNA maturation in Arabidopsis thaliana. Plant Cell Physiol 2020, 61, (6), 1080-1094.

67. Ligas, J.; Pineau, E.; Bock, R.; Huynen, M. A.; Meyer, E. H., The assembly pathway of complex I in Arabidopsis thaliana. Plant J 2019, 97, (3), 447-459.

68. Xiu, Z.; Sun, F.; Shen, Y.; Zhang, X.; Jiang, R.; Bonnard, G.; Zhang, J.; Tan, B. C., EMPTY PERICARP16 is required for mitochondrial nad2 intron 4 cis-splicing, complex I assembly and seed development in maize. Plant $J 2016$.

69. Liu, Y.; He, J.; Chen, Z.; Ren, X.; Hong, X.; Gong, Z., ABA overly-sensitive 5 (ABO5), encoding a pentatricopeptide repeat protein required for cis-splicing of mitochondrial nad2 intron 3, is involved in the abscisic acid response in Arabidopsis. Plant J 2010, 63, (5), 749-65.

70. Hsu, Y. W.; Wang, H. J.; Hsieh, M. H.; Hsieh, H. L.; Jauh, G. Y., Arabidopsis mTERF15 is required for mitochondrial nad2 intron 3 splicing and functional complex I activity. PLoS ONE 2014, 9, (11), e112360.

71. Nakagawa, N.; Sakurai, N., A mutation in At-nMat1a, which encodes a nuclear gene having high similarity to group II Intron maturase, causes impaired splicing of mitochondrial nad4 transcript and altered carbon metabolism in Arabidopsis thaliana. Plant Cell Physiol 2006, 47, (6), 772-783.

72. Keren, I.; Tal, L.; Colas des Francs-Small, C.; Araújo, W. L.; Shevtsov, S.; Shaya, F.; Fernie, A. R.; Small, I.; Ostersetzer-Biran, O., nMAT1, a nuclear-encoded maturase involved in the trans-splicing of nad1 intron 1, is essential for mitochondrial complex I assembly and function. Plant $J$ 2012, 71, (3), 413-426.

73. Perales, M.; Parisi, G.; Fornasari, M. S.; Colaneri, A.; Villarreal, F.; Gonzalez-Schain, N.; Echave, J.; Gomez-Casati, D.; Braun, H. P.; Araya, A.; Zabaleta, E., Gamma carbonic anhydrase like complex interact with plant mitochondrial complex I. Plant Mol Biol 2004, 56, (6), 947-57.

74. Pineau, B.; Layoune, O.; Danon, A.; De Paepe, R., L-galactono-1,4-lactone dehydrogenase is required for the accumulation of plant respiratory complex I. J Biol Chem 2008, 283, (47), 32500-32505.

75. Karpova, O. V.; Kuzmin, E. V.; Elthon, T. E.; Newton, K. J., DifferentialeExpression of alternative oxidase genes in maize mitochondrial mutants. Plant Cell 2002, 14, (12), 3271-3284.

76. Ostersetzer-Biran, O., Respiratory complex I and embryo development. J Exp Bot 2016, 67, (5), 1205-7.

77. Michel, F.; Umesono, K.; Ozeki, H., Comparative and functional anatomy of group II catalytic introns - a review. Gene 1989, $82,(1), 5-30$.

78. Michel, F.; Ferat, J. L., Structure and activities of group-II introns. Annual review of biochemistry 1995, 64, 435-461.

79. Lazowska, J.; Jacq, C.; Slonimski, P. P., Sequence of introns and flanking exons in wild-type and box3 mutants of cytochrome b reveals an interlaced splicing protein coded by an intron. Cell 1980, 22, (2 Pt 2), 333-48.

80. Wang, C.; Fourdin, R.; Quadrado, M.; Dargel-Graffin, C.; Tolleter, D.; Macherel, D.; Mireau, H., Rerouting of ribosomal proteins into splicing in plant organelles. P Natl Acad Sci USA 2020, 117, (47), 29979-29987.

81. Fujii, S.; Small, I., The evolution of RNA editing and pentatricopeptide repeat genes. New Phytol 2011, 191, 37-47.

82. Matsuura, M.; Noah, J. W.; Lambowitz, A. M., Mechanism of maturase-promoted group II intron splicing. EMBO J 2001, 20, (24), 7259-7270.

83. Schertl, P.; Braun, H. P., Respiratory electron transfer pathways in plant mitochondria. Front Plant Sci $2014,5,163$.

84. Senkler, J.; Senkler, M.; Eubel, H.; Hildebrandt, T.; Lengwenus, C.; Schertl, P.; Schwarzländer, M.; Wagner, S.; Wittig, I.; Braun, H.-P., The mitochondrial complexome of Arabidopsis thaliana. Plant J 2017, 89, (6), 1079-1092.

85. Subrahmanian, N.; Remacle, C.; Hamel, P. P., Plant mitochondrial complex I composition and assembly: A review. BBA - Bioenergetics 2016, 1857, (7), 1001-14. 
86. Jacoby, R. P.; Li, L.; Huang, S.; Pong Lee, C.; Millar, A. H.; Taylor, N. L., Mitochondrial composition, function and stress response in plants. J Integr Plant Biol 2012, 54, (11), 887-906.

87. Lee, C. P.; Taylor, N. L.; Millar, A. H., Recent advances in the composition and heterogeneity of the Arabidopsis mitochondrial proteome. Front Plant Sci 2013, 4.

88. Braun, H.-P.; Binder, S.; Brennicke, A.; Eubel, H.; Fernie, A. R.; Finkemeier, I.; Klodmann, J.; König, A.-C.; Kühn, K.; Meyer, E.; Obata, T.; Schwarzländer, M.; Takenaka, M.; Zehrmann, A., The life of plant mitochondrial complex I. Mitochondrion 2014, 19, Part B, (0), 295-313.

89. Klodmann, J.; Sunderhaus, S.; Nimtz, M.; Jänsch, L.; Braun, H.-P., Internal architecture of mitochondrial complex I from Arabidopsis thaliana. Plant cell 2010, 22, (3), 797-810.

90. Soufari, H.; Parrot, C.; Kuhn, L.; Waltz, F.; Hashem, Y., Specific features and assembly of the plant mitochondrial complex I revealed by cryo-EM. Nat Comm 2020, 11, (1), 5195.

91. Maldonado, M.; Padavannil, A.; Zhou, L.; Guo, F.; Letts, J. A., Atomic structure of a mitochondrial complex I intermediate from vascular plants. eLife 2020, 9, e56664.

92. Pinfield-Wells, H.; Rylott, E. L.; Gilday, A. D.; Graham, S.; Job, K.; Larson, T. R.; Graham, I. A., Sucrose rescues seedling establishment but not germination of Arabidopsis mutants disrupted in peroxisomal fatty acid catabolism. Plant J 2005, 43, (6), 86172.

93. Koprivova, A.; des Francs-Small, C. C.; Calder, G.; Mugford, S. T.; Tanz, S.; Lee, B. R.; Zechmann, B.; Small, I.; Kopriva, S., Identification of a pentatricopeptide repeat protein implicated in splicing of intron 1 of mitochondrial nad7 transcripts. J Biol Chem 2010, 285, (42), 32192-9.

94. Colas des Francs-Small, C.; Falcon de Longevialle, A.; Li, Y.; Lowe, E.; Tanz, S. K.; Smith, C.; Bevan, M. W.; Small, I., The Pentatricopeptide Repeat Proteins TANG2 and ORGANELLE TRANSCRIPT PROCESSING439 are involved in the splicing of the multipartite nad5 transcript encoding a subunit of mitochondrial complex I. Plant Physiol 2014, 165, (4), 1409-1416.

95. Weissenberger, S.; Soll, J.; Carrie, C., The PPR protein SLOW GROWTH 4 is involved in editing of nad4 and affects the splicing of nad2 intron 1. Plant Mol Biol 2017, 93, (4-5), 355-368.

96. Zmudjak, M.; Colas des Francs-Small, C.; Keren, I.; Shaya, F.; Belausov, E.; Small, I.; Ostersetzer-Biran, O., mCSF1, a nucleusencoded CRM protein required for the processing of many mitochondrial introns, is involved in the biogenesis of respiratory complexes I and IV in Arabidopsis. New Phytol 2013, 199, (2), 379-394.

97. Cohen, S.; Zmudjak, M.; Colas des Francs-Small, C.; Malik, S.; Shaya, F.; Keren, I.; Belausov, E.; Many, Y.; Brown, G. G.; Small, I.; Ostersetzer-Biran, O., nMAT4, a maturase factor required for nad1 pre-mRNA processing and maturation, is essential for holocomplex I biogenesis in Arabidopsis mitochondria. Plant J 2014, 78, (2), 253-268.

98. Sultan, L. D.; Mileshina, D.; Grewe, F.; Rolle, K.; Abudraham, S.; Głodowicz, P.; Khan Niazi, A.; keren, I.; Shevtsov, S.; Klipcan, L.; Barciszewski, J.; Mower, J. P.; Dietrich, A.; Ostersetzer, O., The reverse-transcriptase/RNA-maturase protein MatR is required for the splicing of various group II introns in Brassicaceae mitochondria. Plant cell 2016, 28, (11), 2805-2829. 